\title{
ARTICLE OPEN \\ Identification of stable adsorption sites and diffusion paths on nanocluster surfaces: an automated scanning algorithm
}

\author{
Tibor Szilvási ${ }^{1,2}$, Benjamin W. J. Chen (iD ${ }^{1,2}$ and Manos Mavrikakis (D) $^{1 *}$
}

The diverse coordination environments on the surfaces of discrete, three-dimensional (3D) nanoclusters contribute significantly to their unique catalytic properties. Identifying the numerous adsorption sites and diffusion paths on these clusters is however tedious and time-consuming, especially for large, asymmetric nanoclusters. Here, we present a simple, automated method for constructing approximate 2D potential energy surfaces for the adsorption of atomic species on the surfaces of 3D nanoclusters with minimal human intervention. These potential energy surfaces fully characterize the important adsorption sites and diffusion paths on the nanocluster surfaces with accuracies similar to current approaches and at comparable computational cost. Our method can treat complex nanoclusters, such as alloy nanoclusters, and accounts for cluster relaxation and adsorbate-induced reconstruction, important for obtaining accurate energetics. Moreover, its highly parallelizable nature is ideal for modern supercomputer architectures. We showcase our method using two clusters: $\mathrm{Au}_{18}$ and $\mathrm{Pt}_{55}$. For $\mathrm{Au}_{18}$, diffusion of atomic hydrogen between the most stable sites occurs via non-intuitive paths, underlining the necessity of exploring the complete potential energy surface. By enabling the rapid and unbiased assessment of adsorption and diffusion on large, complex nanoclusters, which are particularly difficult to handle manually, our method will help advance materials discovery and the rational design of catalysts.

npj Computational Materials (2019)5:101

; https://doi.org/10.1038/s41524-019-0240-x

\section{INTRODUCTION}

The use of metal nanoclusters in catalytic processes has steadily increased due to their higher dispersion and often times higher intrinsic activities compared with larger metal nanoparticles. ${ }^{1-4}$ This increased catalytic activity is mainly attributed to the presence of stable low-coordination sites, ${ }^{5,6}$ although other effects, such as quantum-size effects, ${ }^{7,8}$ surface-tension-induced strain, ${ }^{9}$ and support-metal interactions ${ }^{10,11}$ can also be relevant. Due to these effects, the reactivity of nanoclusters, including their preferred reaction pathways and intermediates, can vary greatly with small changes in their size down to the addition or removal of single atoms. $^{1-4}$ The variability in the reactivity of nanoclusters hinders theoretical efforts to rapidly characterize them and screen for the ones with optimal catalytic properties, whereas similar efforts are already well under way for extended solid surfaces ${ }^{12,13}$ and for bulk materials as part of the Materials Genome Initiative. ${ }^{14-16}$

To characterize the catalytic properties of nanoclusters, the computational catalysis community typically performs manual guess-and-optimize calculations to systematically probe the reactivity of the unique site types on these clusters. Although the computational and human workload for manual exploration of small clusters is manageable, comprehensive investigations of larger, more complex nanoclusters (e.g., alloy nanoclusters) requires extensive and tedious human effort. In these situations, the possibility of missing potentially important adsorption sites also increases rapidly.

Despite the significant drawbacks of traditional guess-andoptimize calculations, there are currently few methods to probe the surface reactivity of clusters in an automated way. One approximate technique for probing adsorption on cluster surfaces is the generalized coordination number, ${ }^{17}$ which can predict adsorption energies on monometallic surfaces, but cannot be easily extended to describe alloys. Another recently developed technique that does treat alloy surfaces is the Orbitalwise Coordination Number. ${ }^{18}$ Both techniques however are unable to account for adsorbate-induced cluster relaxation and reconstruction, an important phenomenon that can greatly increase the reactivity of clusters. ${ }^{1,19}$ Additionally, these techniques are unable to probe other aspects of surface reactivity such as diffusion of adsorbed intermediates on nanocluster surfaces. Recently, machine learning has emerged as another option for studying nanoclusters, ${ }^{20,21}$ however, automatic generation of the large amounts of high-quality required training data remains a problem. $^{22}$

Potential energy surfaces (PESs), on the other hand, fully characterize the reactivity of a surface, from binding strengths of adsorbates to their barriers for diffusion. They can account for adsorbate-induced reconstructions, are generalizable to alloy surfaces, and greatly reduce the possibility of missing potential adsorption sites and diffusion paths. Furthermore, constructing PESs requires minimal human intervention and thus reduces the human workload; generating such PESs can be easily automated as it involves simply repeating the evaluation of the energy of a system along multiple points of its PES. Because PESs are rich in information, researchers increasingly employ them in catalysisrelated problems. ${ }^{23,24}$ Until now however, PESs have mainly been used to study two-dimensional (2D) surfaces such as extended slab models.

Here, we present a simple way to generate PESs for 3D cluster surfaces, called here forth the Automated Cluster Surface Scanning (ACSS) method, and demonstrate the accuracy and efficiency of our method by probing the PES for adsorption of atomic $\mathrm{H}$ on $\mathrm{Au}_{18}$ and $\mathrm{Pt}_{55}$ nanoclusters. We chose these clusters since previous experimental results showed that small gold and platinum clusters are active in various catalytic processes involving adsorbed surface $\mathrm{H}^{25-29}$ Our contribution is structured as follows: 
first, we demonstrate via comparisons with standard manually performed (MP) calculations that our ACSS methodology identifies the most important stable adsorption sites and diffusion paths connecting any two of these sites on a given metal nanocluster surface. Second, we quantify the errors introduced by ACSS and assess the validity of the assumptions in ACSS. Lastly, we compare the computational cost of the ACSS method with that of MP calculations.

\section{RESULTS}

\section{Scanning algorithm}

Computing the PES of an adsorbate binding on a nanocluster surface requires calculating the total energy of the nanocluster + adsorbate system at each point on the nanocluster surface. Figure 1 illustrates the main idea behind the ACSS method, which we designed to do exactly this. After selecting a well-defined point within the nanocluster, such as its center of mass (CoM), we can parameterize any point on its surface (green vectors, Fig. 1) with three spherical coordinates: (i) $a$, the azimuthal angle $\left(0^{\circ} \leq a \leq\right.$ $\left.180^{\circ}\right)$; (ii) $\beta$, the polar angle $\left(0^{\circ} \leq \beta<360^{\circ}\right)$; and (iii) $R$, the radial distance $(R>0)$, which is the distance between the adsorbate and the cluster's CoM. Placing the adsorbate at a fixed $\alpha$ and $\beta$, and optimizing all other degrees of its freedom (for a monoatomic adsorbate, this is equivalent to optimizing $R$ only), we can obtain the energy of the system at any given $(a, \beta)$ coordinate pair. By systematically varying $a$ and $\beta$, thus "scanning" across the cluster surface, we can formally construct the 2D PES of the cluster surface as a function of $a$ and $\beta$ for any convex structure. The surface of a concave structure can be considered as a united surface of multiple convex parts, each of which can be handled as described below. Thus, for the sake of simplicity, we do not investigate concave structures in this paper.

Our implementation of the ACSS method involves some approximations. First, the PES is constructed by sampling a finite number of points. We use spline interpolation to create a smoother PES, which introduces errors at points where we did not explicitly calculate the energy. By increasing the number of

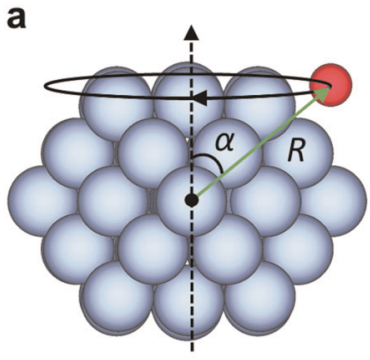

Side view

b

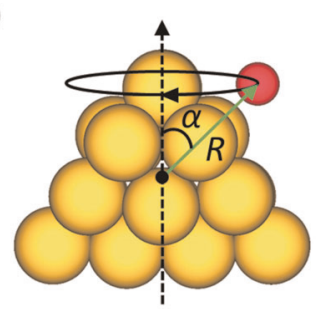

Side view

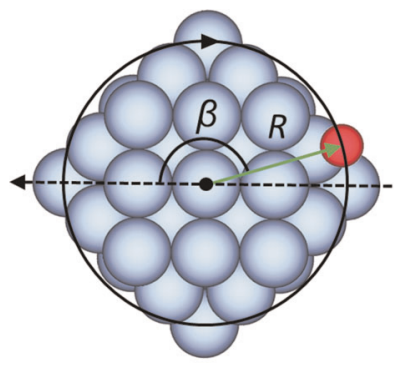

Top view

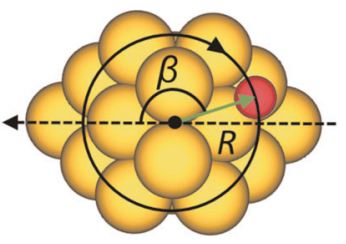

Top view
Fig. 1 Illustration of the Automated Cluster Surface Scanning (ACSS) algorithm. Parameterization of the $\mathbf{a} \mathrm{Pt}_{55}$ and $\mathbf{b} \mathrm{Au}_{18}$ nanocluster surfaces in the ACSS method, using the center of mass (black dot) as the origin. Color code: gold $-\mathrm{Au}$, blue $-\mathrm{Pt}$, red $-\mathrm{H}$. $a$ and $\beta$ run between $0-180^{\circ}$ and $0-360^{\circ}$, respectively sampled points however, we can systematically reduce this interpolation error. In this study, we sample the PES using simple, equidistant meshes with fixed intervals in $(\alpha, \beta)$. Note that these equidistant meshes in $(\alpha, \beta)$ space are not equidistant in real space and thus lead to oversampling of real space regions when $a$ is close to $0^{\circ}$ or $180^{\circ}$; one may wish to use more complex meshes that can sample real space uniformly to save on computational cost.

Secondly, we must fix several degrees of freedom of the nanocluster to preserve the integrity of our chosen coordinate system during the constrained optimization of the adsorbate's position. If we allow the entire nanocluster to relax while the $a$ and $\beta$ coordinates of the adsorbate are fixed, the nanocluster may rotate or translate relative to the adsorbate to lower the overall energy of the system. This is ultimately equivalent to an unwanted shift in the $a$ and $\beta$ coordinates of the adsorbate. For a Cartesian coordinate system, at least three atoms have to be fixed to avoid the relative rotation and translation motion of the cluster. This introduces a second source of error by neglecting part of the relaxation energy of the entire cluster.

\section{Example systems}

To verify the accuracy of the ACSS method, we used it to study atomic hydrogen $(\mathrm{H})$ adsorption on two clusters: (i) $\mathrm{Pt}_{55}, \mathrm{a}$ medium-sized cluster with two shells of Pt atoms; and (ii) $\mathrm{Au}_{18}, \mathrm{a}$ small cluster with just one shell of Au atoms (i.e., all atoms are on the surface). The structure of the cluster largely determines which atoms to fix: because relaxation mainly affects atoms close to the adsorbate, which are the surface atoms of the nanocluster, it is logical to fix core atoms or atoms far from catalytically interesting regions of the metal cluster to minimize errors due to the neglect of relaxation effects. In the case of $\mathrm{Pt}_{55}$, we fixed the 13 core atoms and relaxed all the 42 outer shell atoms, which is analogous to relaxing the upper layers of a slab, a common technique to account for relaxation effects in slab calculations. ${ }^{30}$ Using this approach, we expect to capture most relaxation effects. In case of $\mathrm{Au}_{18}$ we fixed the 4 "core" atoms with the largest coordination numbers, as they are expected to relax to a smaller extent compared with the more highly undercoordinated atoms in the cluster (Fig. 2). Yet, these atoms are still part of the surface and directly in contact with the adsorbates; we thus expect to capture less relaxation effects compared with $\mathrm{Pt}_{55}$. Both clusters were first fully relaxed before any atoms were fixed.

As reference calculations to compare the results from our ACSS method with, we also explored all local minima and diffusion paths manually using unconstrained optimization and climbing image nudged elastic band (Cl-NEB) calculations. In these calculations, all adsorbate and cluster atoms were relaxed. For brevity, we herein refer to results obtained with these methods as "Manually-Performed calculations" (MP), and results obtained from the PESs constructed via the ACSS method as "ACSS calculations".

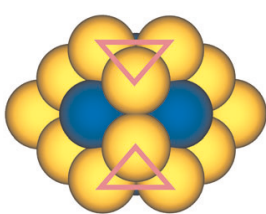

Top view

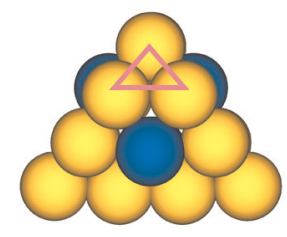

Side view 1

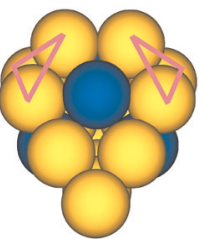

Side view 2
Fig. 2 Atom types in $\mathrm{Au}_{18}$. Fixed (blue) and relaxed atoms (gold) of $\mathrm{Au}_{18}$ for the Automated Cluster Surface Scanning (ACSS) method. Side view 2 is rotated by $90^{\circ}$ with respect to side view 1 . Pink triangles mark two triangular ensembles of Au atoms, which may provide a useful reference frame for the reader 
Potential energy surfaces

By leveraging the symmetry of the clusters, only a portion of the cluster surfaces needs to be sampled to construct the full PESs. For $\mathrm{Pt}_{55}$, we sampled from $a=0-90^{\circ}$ and $\beta=0-44^{\circ}$ with a mesh grid of $2^{\circ}$ intervals, and for $\mathrm{Au}_{18}$, we sampled from $\alpha=0-180^{\circ}$ and $\beta=$ $0-87^{\circ}$ with a mesh grid of $3^{\circ}$ intervals. These intervals correspond to a maximum real space distance of $\sim 0.2 \AA$ between points; the axes of reference for $a=0^{\circ}$ and $\beta=0^{\circ}$ are shown in Fig. 1. The full PES was then generated by applying the relevant symmetry operations, and finally spline interpolation onto a finer mesh grid with $0.5^{\circ}$ intervals to obtain a relatively continuous PES. Our choices of a symmetric origin-the center of mass, and a symmetric arrangement of the fixed metal atoms, were instrumental in fully utilizing the symmetry of the cluster.

Our PESs are well-converged with respect to the sampling mesh: coarser grids with $1 / 4$ and $1 / 16$ the mesh points of the original grids resulted in interpolation errors of just 0.01 and $0.04 \mathrm{eV}$, respectively, for both $\mathrm{Au}_{18}$ and $\mathrm{Pt}_{55}$ (See Supplementary Discussion).

The PESs obtained from the ACSS method can be represented in multiple formats, the most common of which is a contour plot, shown in Fig. $3 \mathrm{a}, \mathrm{b}$ for $\mathrm{Pt}_{55}$ and $\mathrm{Au}_{18}$, respectively. Due to space constraints, we only show a portion of the PESs, enough to capture all unique high-symmetry sites; the full PESs can be found in Supplementary Figs. 3 and 4. To focus on the relative stability of various adsorption sites, all energies reported are relative to the global minimum for each system: the most stable adsorption site has an energy of $0 \mathrm{eV}$ and less stable sites have positive energies. Definitions of the various site types can be found in Supplementary Tables 4 and 7 for $\mathrm{Au}_{18}$ and $\mathrm{Pt}_{55}$, respectively.

The benefits of using PESs to study adsorption on cluster surfaces are apparent from the contour plots (Fig. 3a, b). One can readily assess the positions of stable adsorption sites (local minima on the PES), and view the minimum energy diffusion paths and associated transition states (first order saddle points on the PES), which may not be easily found with manually performed calculations. For example, on $\mathrm{Au}_{18}$ (Fig. 3b) two transition states exist between the $b 2\left(a=45^{\circ}, \beta=110^{\circ}\right)$ and $b 1 \quad\left(a=0^{\circ}, \beta=\right.$ $0-180^{\circ}$; all $\beta$ values are equivalent at $a=0^{\circ}$ ) site types. The existence of these multiple diffusion pathways is easy to overlook: we did not find them while performing the MP calculations, only realizing their existence after we generated and analyzed the PESs.

Although the PESs in Fig. 3 are largely smooth and continuous, there are areas of relatively steep energy changes, identifiable by the areas shaded in black in the PESs, which are due to the overlapping of multiple contour lines in a small region. One such area is close to the bottom of the $f 5$ site type on $\operatorname{Au}_{18}\left(a=80^{\circ}, \beta=\right.$ $180^{\circ}$; near the middle of Fig. 3b). These areas are caused by adsorbate-induced reconstruction of the cluster, and are especially prominent for $\mathrm{Au}_{18}$ as it is smaller and is thus more prone to reconstruction compared with the larger $\mathrm{Pt}_{55}$ cluster.

As the structure of nanoclusters can be complex, the 2D contour plots of their PESs can be difficult to interpret. We thus present an alternative representation of the PESs of $\mathrm{Au}_{18}+\mathrm{H}$ and $\mathrm{Pt}_{55}+\mathrm{H}$ systems by "projecting" it directly onto the surface of the corresponding metal cluster: the cluster-projected representation (Fig. 3c-f). The cluster-projected representation serves as a "heat map", providing immediate and intuitive information regarding the locations of reactive regions on the cluster. For example, one can immediately tell that the areas marked with pink triangles in Fig. $3 \mathrm{~d}-\mathrm{f}$ bind $\mathrm{H}$ much more strongly than other parts of the $\mathrm{Au}_{18}$ cluster. Additionally, diffusion paths are also readily visualized on the cluster.

\section{Accuracy of the ACSS method}

We quantified the accuracy of the data obtained from our method with respect to standard MP calculations in terms of two metrics
(Table 1): (i) the number of stationary points (minima and transition states) found; and (ii) the errors in the energies of the stationary points and diffusion barriers. A parity plot of the energies of the stationary points from ACSS versus MP calculations is also shown in Fig. 4 (numbers in Supplementary Tables 2 and 3 for $\mathrm{Au}_{18}$ and Supplementary Tables 5 and 6 for $\mathrm{Pt}_{55}$ ).

For $\mathrm{Pt}_{55}$, all 29 minima and transition states found with MP calculations were also found with ACSS calculations. The relative site stability is well-preserved; ACSS calculations predict $b 1$ as the most stable site, and $f$ as the least stable site (Supplementary Table 5), identical to MP calculations.

Quantitatively, for $\mathrm{Pt}_{55}$, there is also excellent agreement between MP and ACSS calculations in terms of the energies of stationary points (Table 1). Both transition states and minima are accurately described by the ACSS calculations, with a small root mean squared error (RMSE) of $0.02 \mathrm{eV}$. Diffusion barriers are slightly better described, with RMSEs of just $0.01 \mathrm{eV}$, likely due to error cancellation as barriers are differences in energies between transition states and minima. A linear least-squares fit on the MP versus ACSS results (Fig. 4a) shows an excellent correlation with a slope of nearly one, and an intercept of close to zero, demonstrating that the systematic errors from the ACSS method are almost negligible when the surface atoms are relaxed, allowing us to capture most of the relaxation energy.

For $\mathrm{Au}_{18}, \mathrm{ACSS}$ calculations again preserve the relative site stability of the different sites well: both MP and ACSS calculations predict the $b 3$ as the most stable site, and $h 6$ as the least stable site (Supplementary Table 2). The ACSS calculations, however, failed to locate one minimum (site b13, Supplementary Table 4), and one of its associated transition states, which we know to exist from the MP calculations. This site was created by adsorbateinduced reconstructions of the cluster that were not captured by the ACSS calculations as they occurred near the four fixed Au atoms. This minimum is however shallow; the barrier for diffusion to a neighboring site $(h 4)$ is $<0.01 \mathrm{eV}(b 13-h 4$, Supplementary Table 3; note that the value in the table $(0.03 \mathrm{eV})$ is the barrier with $h 4$ as the initial state, whereas the barrier with b13 as the initial state is $<0.01 \mathrm{eV}$ ). Therefore, we believe this site is of limited importance for understanding realistic experimental processes, especially after considering zero-point vibrational and finitetemperature effects. Overall, the ACSS method performed remarkably well, capturing $96 \%$ (45 out of 47 ) of the stationary points on $\mathrm{Au}_{18}$.

The RMSE for the ACSS-calculated energies of stationary points on $\mathrm{Au}_{18}$ is $0.05 \mathrm{eV}$, higher than those for $\mathrm{Pt}_{55}(0.02 \mathrm{eV})$ (Table 1). This is because of the larger neglect of relaxation effects in the case of $\mathrm{Au}_{18}$, as all the fixed atoms are on the surface of the cluster. The larger RMSE is also reflected in the weaker linear correlation between the MP and the ACSS results for $\mathrm{Au}_{18}$, which has a slope less than unity, although the intercept remains close to zero (Fig. 4b). Errors in two transition state energies (b6-t4 and b5t4, Supplementary Table 3) contribute largely to this weaker correlation; the RMSE for transition states is $0.06 \mathrm{eV}$, double that the RMSE of $0.03 \mathrm{eV}$ for minima. Excluding these two outlier TSs, the accuracy of ACSS improves greatly: the slope increases to $0.94 \pm 0.04$, the $R^{2}$ value rises to 0.98 , and the RMSE of TSs drops to $0.03 \mathrm{eV}$.

\section{Computational cost}

To evaluate the practical applicability of the ACSS method, we compared the computational cost (in terms of number of geometric optimization iterations) of ACSS calculations (ACSS; Fig. 5) with that of MP calculations (MP Relaxed; Fig. 5) for both the $\mathrm{Au}_{18}+\mathrm{H}$ and $\mathrm{Pt}_{55}+\mathrm{H}$ systems. As fixing atoms reduces the number of degrees of freedom, thereby potentially reducing the computational cost involved and biasing our results in favor of the ACSS calculations, we additionally evaluated the 

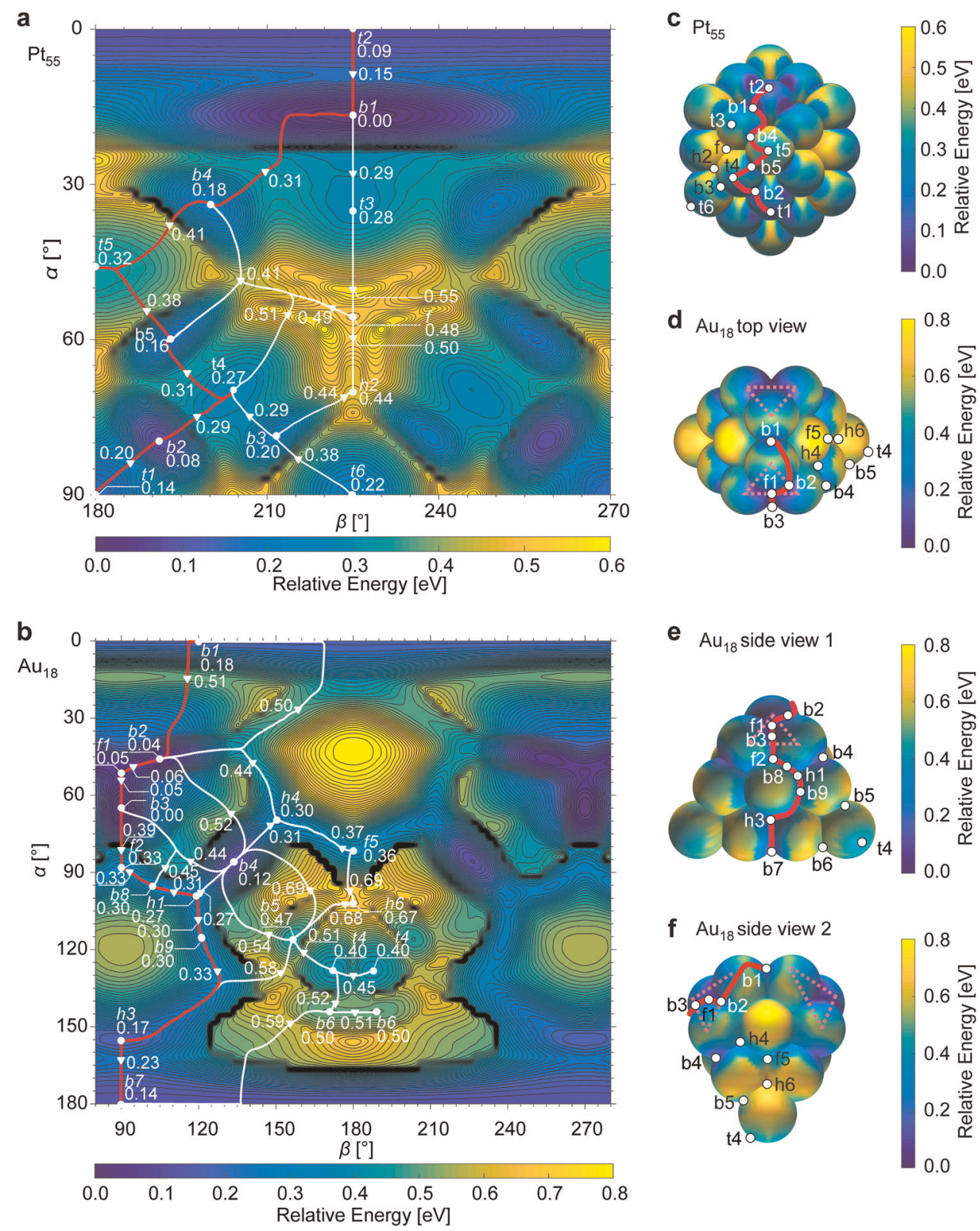

Fig. 3 Representations of potential energy surfaces constructed via the Automated Cluster Surface Scanning (ACSS) method. a, b Contour plots and $\mathbf{c}-\mathbf{f}$ cluster-projected representations for the $(\mathbf{a}, \mathbf{c}) \mathrm{Pt}_{55}+\mathrm{H}$ and $(\mathbf{b}, \mathbf{d}-\mathbf{f}) \mathrm{Au}_{18}+\mathrm{H}$ systems. Energies are relative to the most stable site for each system. White and red lines are the minimum energy paths connecting transition states (white triangles, contour plots only) and minima (white circles and site names). Red lines on the contour plots and the corresponding cluster-projected representations indicate diffusion paths that serve as a frame of reference for the reader. For clarity, only limited ranges of the PESs large enough to show all symmetrically unique sites are shown. Site types are defined in Supplementary Tables 4 and 7 for $\mathrm{Au}_{18}$ and $\mathrm{Pt}_{55}$, respectively. Dotted pink triangles mark the two triangular ensembles of $\mathrm{Au}$ atoms in $\mathrm{Au}_{18}$, identical to those in Fig. 2

computational cost of MP calculations with the same fixed atoms as used in the ACSS calculations (MP Fixed; Fig. 5) to ensure a fair comparison. Details regarding calculations of the computational costs and, for MP calculations, their breakdown into costs of geometry optimization and NEB calculations, can be found in Supplementary Discussion.

Surprisingly, the ACSS calculations for $\mathrm{Au}_{18}$ cost more than that for $\mathrm{Pt}_{55}$, even though $\mathrm{Au}_{18}$ has fewer degrees of freedom with just 14 relaxed atoms whereas $\mathrm{Pt}_{55}$ has 42 relaxed atoms. This is because $\mathrm{Au}_{18}$ has lower symmetry $\left(\mathrm{C}_{2 \mathrm{v}}\right)$ than $\mathrm{Pt}_{55}\left(\mathrm{D}_{4 \mathrm{~h}}\right)$, thus more mesh points are required to map the cluster surface. The cost of the MP calculations for $\mathrm{Au}_{18}$ is, however, less than that for $\mathrm{Pt}_{55}$, due mainly to the lower cost for NEB calculations (Supplementary Table 1), which are known to converge slowly especially with standard optimizers. ${ }^{31,32} \mathrm{Au}_{18}$, with fewer degrees of freedom, is easier for NEB calculations to handle than $\mathrm{Pt}_{55}$. The benefits of reducing the number of degrees of freedom on the cost of NEB calculations are also reflected in the much lower computational cost for the MP Fixed versus MP Relaxed calculations for $\mathrm{Pt}_{55}$ (Fig. 5).

For both $\mathrm{Au}_{18}$ and $\mathrm{Pt}_{55}$, however, the ACSS calculations were less costly than both the MP Relaxed and MP Fixed calculations (Fig. 5). The savings in computational cost were especially large for $\mathrm{Pt}_{55}$ - the ACSS calculations were almost 5 (7) times less costly than the MP Fixed (MP Relaxed) calculations-but less for $\mathrm{Au}_{18}$, where the ACSS calculations cost almost as much as the MP Fixed calculations and were only $\sim 1.5$ times less costly than the MP Relaxed calculations. 
Table 1. Comparison of results from Automated Cluster Surface Scanning (ACSS) calculations and manually-performed (MP) calculations

\begin{tabular}{|c|c|c|c|c|c|c|c|}
\hline & \multicolumn{3}{|c|}{ Number of stationary points ${ }^{a}$} & \multicolumn{4}{|c|}{ Root mean squared errors $[\mathrm{eV}]$} \\
\hline & Minima & Transition states & Overall & Minima & Transition states & Overall & Diffusion barriers \\
\hline $\mathrm{Pt}_{55}$ & $13(13)$ & $16(16)$ & $29(29)$ & 0.02 & 0.02 & 0.02 & 0.01 \\
\hline $\mathrm{Au}_{18}$ & $17(18)$ & $28(29)$ & $45(47)$ & 0.03 & 0.06 & 0.05 & 0.05 \\
\hline
\end{tabular}

a

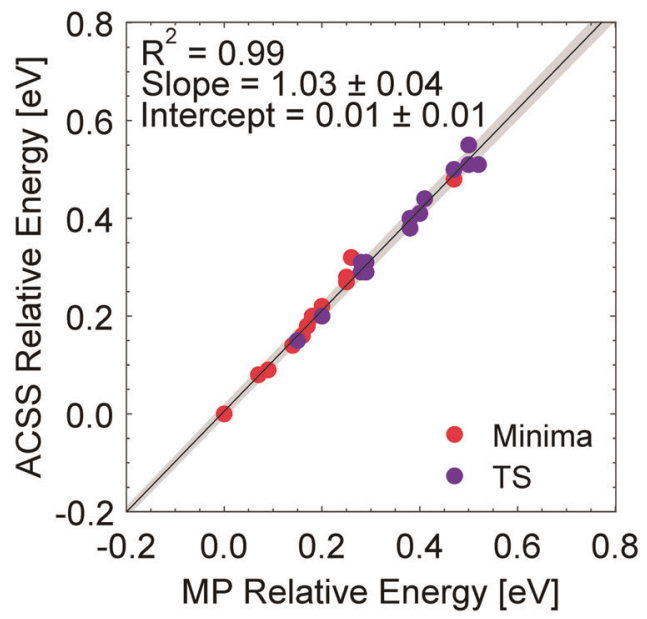

b $\mathrm{Au}_{18}$

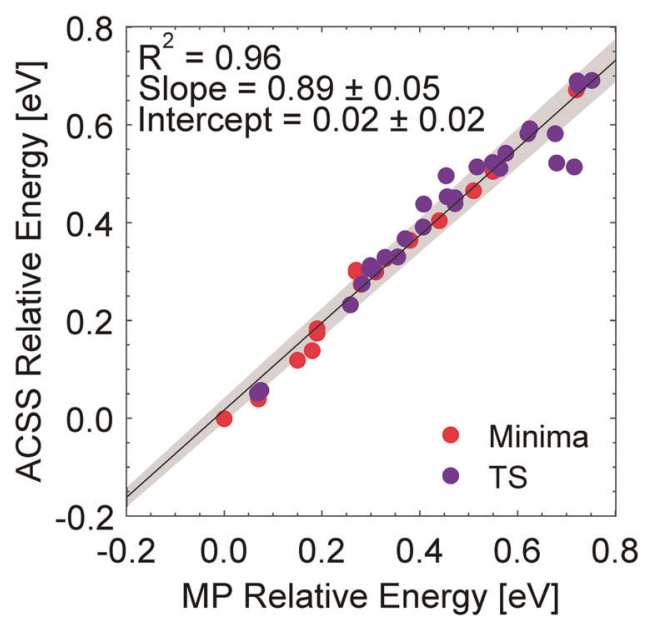

Fig. 4 Accuracy of the Automated Cluster Surface Scanning (ACSS) method. Parity plots of the energies of minima and transition states, relative to the global minimum for each system, derived from ACSS and manually performed (MP) calculations for the (a) $\mathrm{Pt}_{55}+\mathrm{H}$ and (b) $\mathrm{Au}_{18}+\mathrm{H}$ systems. Full lines represent linear fits to the data, and gray regions represent the $95 \%$ confidence intervals for the fits

Several thousand independent $\mathrm{E}(\alpha, \beta)$ calculations are usually required to compute a PES, which may feel computationally prohibitive. The ACSS method, however, ends up being less costly than MP calculations due to its ability to locate transition states without performing explicit NEB calculations, which are much less

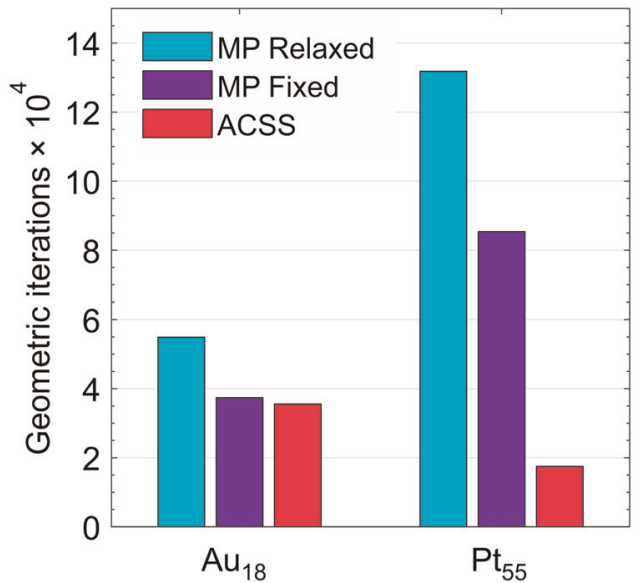

Fig. 5 Computational cost analysis for the Automated Cluster Surface Scanning (ACSS) method. Total computational cost, in terms of geometric optimization iteration steps, of identifying all minima and transition states for manually performed (MP) and Automated Cluster Surface Scanning (ACSS) calculations for the $\mathrm{Au}_{18}+\mathrm{H}$ and $\mathrm{Pt}_{55}+\mathrm{H}$ systems. For MP Relaxed calculations, all atoms were relaxed during optimization. For MP Fixed calculations, the same atoms that were fixed in ACSS calculations were fixed

efficient than geometric optimizations: for our systems, 96-98\% of the computational cost of MP calculations come from NEB calculations (Supplementary Table 1).

The computational cost of the ACSS method can be lowered even further by using a coarser sampling mesh initially and gradually refining the mesh in regions of interest, such as where energies change rapidly, so that the high accuracy required in these regions is preserved. This requires some human intervention but may reduce the computational workload by up to an order of magnitude. For a discussion of the accuracy of coarser meshes, see Supplementary Discussion.

\section{DISCUSSION}

Our results show that the ACSS calculations are of similar accuracy to the MP calculations in terms of finding minima and transition states, and in terms of their description of the energetic landscape. Overall, the ACSS method found $97 \%$ (74 out 76 ) of stationary points, with errors of $\sim 0.05 \mathrm{eV}$-much smaller than those normally associated with DFT calculations $(\sim 0.2 \mathrm{eV}) .^{33}$ We also demonstrated that the computational cost of ACSS calculations is much less than that of MP calculations for $\mathrm{Pt}_{55}$ and similar to that of MP calculations for $\mathrm{Au}_{18}$.

With only a slight sacrifice in accuracy $(\sim 0.05 \mathrm{eV})$, the ACSS method brings several significant advantages over the MP calculations. Firstly, it does not depend on the intuition of the 
researcher and is thus unbiased and reproducible. This is desirable especially for cluster systems that are highly complex. For such systems, it is easy to miss possible adsorption sites or diffusion pathways (as we experienced in the case of $\mathrm{Au}_{18}$; see Results) and tempting to focus only on certain site types, for example, those on the most undercoordinated atoms as they are usually thought to be more reactive. With the ACSS method however, all sites and diffusion pathways can be captured, within the accuracy of our PES.

The $\mathrm{Au}_{18}+\mathrm{H}$ system is one example of a system with nonintuitive diffusion paths. For example, consider the diffusion of $\mathrm{H}$ between the two most stable $b 3$ sites on $\mathrm{Au}_{18}$. Based on intuition alone, it is tempting to think that the shortest diffusion path (red arrow; Fig. 6) is also the most energetically favorable. This path entails a maximum barrier of $0.52 \mathrm{eV}$ with respect to the global minimum (as calculated by the MP method), and involves seven sites going directly over the top of the cluster. The most energetically favorable pathway, however, with a maximum barrier of $0.41 \mathrm{eV}$ with respect to the global minimum, involves 13 sites going around the cluster (green arrow; Fig. 6), and was found easily using our ACSS-constructed PES. The full pathway on the contour-plot representation is shown in Supplementary Fig. 3. This result underlines that diffusion can be a complex process even for a small cluster such as $\mathrm{Au}_{18}$. Trying to elucidate minimum energy diffusion pathways on large nanoclusters by hand is not only tedious but can also lead to missing complicated but energetically relevant diffusion paths. For completeness, the minimum energy diffusion pathways between the most stable sites on $\mathrm{Pt}_{55}$ are included in Supplementary Figs. 4 and 5.

Secondly, calculations of each point on the PES are completely independent of each other, making them highly parallelizable and efficiently executable on supercomputers by distributing the workload among as many nodes or cores as needed. By exploiting the massively parallel nature of the ACSS method on supercomputers, the timeframe of a project-from preparing the first calculation to completing the last-can be greatly reduced. Anecdotally, we required less than 1 day to finish all ACSS calculations for the $\mathrm{Au}_{18}+\mathrm{H}$ system (1800 grid points) on the National Energy Research Scientific Computing Center's (NERSC) Cori supercomputer by parallelizing our calculations across 200 Knights Landing (KNL) nodes. Each node was responsible for the evaluation of a single grid point at a time and was repurposed to evaluate the next uncalculated grid point when the previous calculation finished. The MP calculations, however, required several weeks to complete on the same supercomputer, mainly
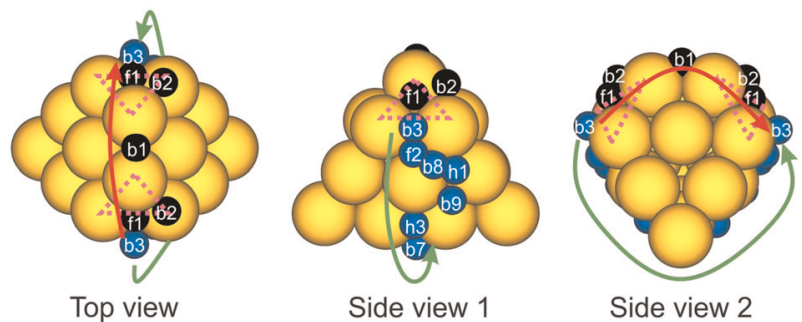

Fig. 6 Diffusion paths of atomic $\mathrm{H}$ on $\mathrm{Au}_{18}$. Red arrows indicate the spatially shortest diffusion path between the two most stable b3 sites, which has an overall barrier of $0.52 \mathrm{eV}$ with respect to the global minimum. Green arrows indicate the most energetically favorable diffusion path, which has an overall barrier of $0.41 \mathrm{eV}$ with respect to the global minimum. Blue and black spheres represent stable $\mathrm{H}$ adsorption sites on the green and red diffusion paths, respectively; gold spheres represent Au atoms. Side view 2 is rotated by $90^{\circ}$ with respect to side view 1 . Dotted pink triangles mark the two triangular ensembles of $\mathrm{Au}$ atoms in $\mathrm{Au}_{18}$, identical to those in Fig. 2 due to the large number of calculations that had to be manually checked and resubmitted if they did not converge or failed.

Small clusters are however a limitation of the ACSS method. As a large fraction of the atoms in these clusters must be fixed in order to prevent rotation and translation of the cluster, a significant portion of the relaxation effects, such as adsorbate-induced reconstruction of the cluster, may be neglected. Such relaxation effects can be important as they affect adsorption energies and may create local minima, as evident from our results showing that $\mathrm{Au}_{18}$ is described less accurately than $\mathrm{Pt}_{55}$. We note, however, that fixing the center of mass in the calculations, combined with larger unit cells and tighter convergence criteria to reduce the drift present in the calculated forces, would help inhibit translation and rotation of the cluster. This may allow for decreasing the fraction of fixed atoms needed for the ACSS methodology, in turn increasing the accuracy of our approach for smaller clusters.

Given the above strengths and limitations of the ACSS method, we expect it to be highly suited for studying large, non-symmetric clusters. Such clusters are frequently found to be very stable despite their low symmetry. ${ }^{34-36}$ Their non-trivial geometries however make them challenging targets for traditional MP computational strategies, which depend heavily on symmetry to reduce the complexity of the system and to keep the mental and computational burden tractable. For large clusters we can also relax multiple shells of atoms, thus allowing us to accurately capture most of the relaxation effects.

Yet, we believe that even for small clusters where the ACSS method is less accurate, or even when very high levels of accuracy are desired, the ACSS method can still be useful as a prescreening approach to quickly identify important minima and diffusion pathways that standard chemical intuition may miss. The geometries from the ACSS method can also be used as excellent initial guesses for follow-up calculations to save computational time; for example, good initial guesses of diffusion pathways, essential for fast convergence of NEB calculations, can be obtained from the minimum energy paths of the PES constructed from the ACSS method. ${ }^{37}$

In the future, we envision that all tasks involved in ACSS calculations and the analysis of the resulting PESs, such as the determination of minima and the initialization of the string method for locating diffusion pathways, can be fully automated. Our method may also be extended to probe recombination reactions and eventually full mechanisms by fixing an adsorbate on the cluster and scanning across the cluster with a second adsorbate. Entropies, vibrational frequencies, and pre-exponential factors for diffusion events may also be obtainable from the PES. ${ }^{23}$ The automated collection of all thermodynamic and kinetic data, when interfaced with codes to perform kinetic modeling, can then enable high-throughput screening of both monometallic and alloy clusters for catalysis and materials science

In summary, we presented an automated, highly parallelizable approach-the ACSS method-to generate approximate 2D PESs of 3D nanocluster surfaces. These PESs fully characterize the stable adsorption sites and diffusion paths of adsorbates on the surfaces of nanoclusters with minimal human workload. Using the test cases of atomic $\mathrm{H}$ adsorption and diffusion on $\mathrm{Au}_{18}$ and $\mathrm{Pt}_{55}$, the ACSS method qualitatively and quantitatively reproduced results obtained by traditional MP calculations at similar or reduced computational cost. Importantly, the realized gains in computational cost increase dramatically with cluster size, a regime exactly where human intuition and detailed accounting of sites and paths would be more likely to fail.

The ACSS method eliminates tedious, repetitive tasks, such as the probing of stable adsorption sites and diffusion barriers. Additionally, they remove user biases and reduce the likelihood of missed potentially important adsorption sites or diffusion paths. Due to the generalizability of our method, we envision that it will serve as a foundation for enabling the automated exploration of 
complex surfaces of large model nanoclusters. Such nanoclusters have technological applications not only in catalysis, as showcased in this work, but also in chemistry and materials science, for example, for analyzing the diffusion of adsorbates on the surfaces and grain boundaries of complex battery cathode materials. In doing so, we hope that our method will greatly accelerate the discovery of new materials and the rational design of catalysts.

\section{METHODS}

All calculations were performed using the PW91 exchange-correlation functional ${ }^{38}$ with projector augmented wave (PAW) pseudopotentials, 39,40 as implemented in the Vienna Ab initio Simulation Package (VASP). ${ }^{41,42}$ The valence electron wave functions were expanded using a plane wave basis set with an energy cutoff of $400 \mathrm{eV}$. The Brillouin zone was sampled at the Gamma point only. To accelerate electronic convergence, the Fermi surface was treated with Gaussian smearing with a width of $0.1 \mathrm{eV}$. The resulting energies were extrapolated to zero width. To avoid interactions with periodic images, at least $15 \AA$ of vacuum was introduced between images in all three directions.

The truncated tetrahedron and cuboctahedron structures were used to model $\mathrm{Au}_{18}$ (Supplementary Fig. 1) and $\mathrm{Pt}_{55}$ (Supplementary Fig. 2), respectively. These geometries are highly stable and commonly used in theoretical studies. ${ }^{43,44}$ All structures were relaxed until the Hellmann-Feynman forces acting on each atom were $<0.02 \mathrm{eV} \AA^{-1}$. The optimized coordinates of the naked $\mathrm{Au}_{18}$ and $\mathrm{Pt}_{55}$ clusters are given in Supplementary Tables 8 and 9 , respectively. Transition states were obtained with the Climbing-Image Nudged Elastic Band (CI-NEB) method for manually performed calculations using the force criterion mentioned earlier. ${ }^{45}$ All stationary points were confirmed by a normal-mode analysis.

Minimum energy pathways and their associated saddle points, as well as minima on our constructed PESs were identified using the zerotemperature String (ZTS) method. ${ }^{46,47}$

\section{DATA AVAILABILITY}

The data supporting the findings of this study are available from the corresponding author upon reasonable request.

\section{CODE AVAILABILITY}

The code used in this study is available from the corresponding author upon reasonable request.

Received: 15 June 2019; Accepted: 3 October 2019; Published online: 25 October 2019

\section{REFERENCES}

1. Crampton, A. S. et al. Structure sensitivity in the nonscalable regime explored via catalysed ethylene hydrogenation on supported platinum nanoclusters. Nat. Commun. 7, 10389 (2016)

2. Vajda, S. et al. Subnanometre platinum clusters as highly active and selective catalysts for the oxidative dehydrogenation of propane. Nat. Mater. 8, 213-216 (2009).

3. Kaden, W. E., Wu, T., Kunkel, W. A. \& Anderson, S. L. Electronic structure controls reactivity of size-selected $\mathrm{Pd}$ clusters adsorbed on $\mathrm{TiO}_{2}$ surfaces. Science 326, 826-830 (2009).

4. Tyo, E. C. \& Vajda, S. Catalysis by clusters with precise numbers of atoms. Nat. Nanotechnol. 10, 577-588 (2015).

5. Honkala, K. et al. Ammonia synthesis from first-principles calculations. Science 307, 555-558 (2005).

6. Lopez, N. On the origin of the catalytic activity of gold nanoparticles for lowtemperature CO oxidation. J. Catal. 223, 232-235 (2004).

7. Kleis, J. et al. Finite size effects in chemical bonding: from small clusters to solids. Catal. Lett. 141, 1067-1071 (2011).

8. Li, L. et al. Investigation of catalytic finite-size-effects of platinum metal clusters. J. Phys. Chem. Lett. 4, 222-226 (2013).

9. An, W. \& Liu, P. Size and shape effects of Pd@Pt core-shell nanoparticles: unique role of surface contraction and local structural flexibility. J. Phys. Chem. C 117, 16144-16149 (2013).
10. Yan, Z., Chinta, S., Mohamed, A., Fackler, J. P. \& Goodman, D. W. The role of Fcenters in catalysis by Au supported on MgO. J. Am. Chem. Soc. 127, 1604-1605 (2005)

11. Yoon, B. et al. Charging effects on bonding and catalyzed oxidation of $\mathrm{CO}$ on $\mathrm{Au}_{8}$ clusters on MgO. Science 307, 403-407 (2005).

12. Himanen, L., Rinke, P. \& Foster, A. S. Materials structure genealogy and highthroughput topological classification of surfaces and 2D materials. npj Comput. Mater. 4, 1-10 (2018).

13. Montoya, J. H. \& Persson, K. A. A high-throughput framework for determining adsorption energies on solid surfaces. npj Comput. Mater. 3, 14 (2017).

14. de Pablo, J. J. et al. New frontiers for the materials genome initiative. npj Comput. Mater. 5, 41 (2019).

15. Xiong, W. \& Olson, G. B. Cybermaterials: materials by design and accelerated insertion of materials. npj Comput. Mater. 2, 15009 (2016).

16. Sumpter, B. G., Vasudevan, R. K., Potok, T. \& Kalinin, S. V. A bridge for accelerating materials by design. npj Comput. Mater. 1, 15008 (2015).

17. Martínez, J. I. et al. Fast prediction of adsorption properties for platinum nanocatalysts with generalized coordination numbers. Angew. Chem. - Int Ed. 53, 8316-8319 (2014).

18. Ma, X. \& Xin, H. Orbitalwise coordination number for predicting adsorption properties of metal nanocatalysts. Phys. Rev. Lett. 118, 36101 (2017).

19. Häkkinen, H., Abbet, S., Sanchez, A., Heiz, U. \& Landman, U. Structural, electronic, and impurity-doping effects in nanoscale chemistry: supported gold nanoclusters. Angew. Chem. - Int Ed. 42, 1297-1300 (2003).

20. Jäger, M. O. J., Morooka, E. V., Federici Canova, F., Himanen, L. \& Foster, A. S. Machine learning hydrogen adsorption on nanoclusters through structural descriptors. npj Comput. Mater. 4, 37 (2018).

21. Jennings, P. C., Lysgaard, S., Hummelshøj, J. S., Vegge, T. \& Bligaard, T. Genetic algorithms for computational materials discovery accelerated by machine learning. npj Comput. Mater. 5, 46 (2019).

22. Ramprasad, R., Batra, R., Pilania, G., Mannodi-Kanakkithodi, A. \& Kim, C. Machine learning in materials informatics: recent applications and prospects. npj Comput. Mater. 3, 54 (2017)

23. García-Diéguez, M., Hibbitts, D. D. \& Iglesia, E. Hydrogen chemisorption isotherms on platinum particles at catalytic temperatures: Langmuir and two-dimensional gas models revisited. J. Phys. Chem. C. 123, 8447-8462 (2019).

24. Garrison, B. J. \& Srivastava, D. Potential energy surfaces for chemical reactions at solid surfaces. Annu Rev. Phys. Chem. 46, 373-394 (1995).

25. Fu, Q., Saltsburg, H. \& Flytzani-Stephanopoulos, M. Active nonmetallic Au and Pt species on ceria-based water-gas shift catalysts. Science 301, 935-8 (2003).

26. Ojeda, M. et al. Kinetically relevant steps and $\mathrm{H}_{2} / \mathrm{D}_{2}$ isotope effects in FischerTropsch synthesis on Fe and Co catalysts. J. Phys. Chem. C. 114, 19761-19770 (2010).

27. Bulushev, D. A., Beloshapkin, S. \& Ross, J. R. H. Hydrogen from formic acid decomposition over Pd and Au catalysts. Catal. Today 154, 7-12 (2010).

28. Yu, W., Mullen, G. M., Flaherty, D. W. \& Mullins, C. B. Selective hydrogen production from formic acid decomposition on $\mathrm{Pd}-\mathrm{Au}$ bimetallic surfaces. J. Am. Chem. Soc. 136, 11070-11078 (2014).

29. Singh, S. et al. Formic acid decomposition on Au catalysts: DFT, microkinetic modeling, and reaction kinetics experiments. AIChE J. 60, 1303-1319 (2014).

30. Sholl, D. S. \& Steckel, J. A. Density Functional Theory. (John Wiley \& Sons, Inc., 2009). https://doi.org/10.1002/9780470447710.

31. Bohner, M. U., Meisner, J. \& Kästner, J. A quadratically-converging nudged elastic band optimizer. J. Chem. Theory Comput 9, 3498-3504 (2013).

32. Koistinen, O.-P., Dagbjartsdóttir, F. B., Ásgeirsson, V., Vehtari, A. \& Jónsson, H. Nudged elastic band calculations accelerated with Gaussian process regression. J. Chem. Phys. 147, 152720 (2017).

33. Norskov, J. K., Abild-Pedersen, F., Studt, F. \& Bligaard, T. Density functional theory in surface chemistry and catalysis. Proc. Natl Acad. Sci. USA 108, 937-943 (2011).

34. Pande, S. et al. Structural evolution of core-shell gold nanoclusters: $\mathrm{Au}_{\mathrm{n}}{ }^{-} \mathrm{(n}=$ 42-50). ACS Nano 10, 10013-10022 (2016).

35. Häkkinen, H. Atomic and electronic structure of gold clusters: understanding flakes, cages and superatoms from simple concepts. Chem. Soc. Rev. 37, 1847 (2008).

36. Piotrowski, M. J. et al. Theoretical Study of the Structural, Energetic, and Electronic Properties of 55-Atom Metal Nanoclusters: A DFT Investigation within van der Waals Corrections, Spin-Orbit Coupling, and PBE $+U$ of 42 Metal Systems. J. Phys. Chem. C. 120, 28844-28856 (2016).

37. Smidstrup, S., Pedersen, A., Stokbro, K. \& Jónsson, H. Improved initial guess for minimum energy path calculations. J. Chem. Phys. 140, 214106 (2014).

38. Perdew, J. P. \& Wang, Y. Accurate and simple analytic representation of the electron-gas correlation energy. Phys. Rev. B 45, 13244-13249 (1992).

39. Blöchl, P. E. Projector augmented-wave method. Phys. Rev. B 50, 17953-17979 (1994). 
40. Kresse, G. \& Joubert, D. From ultrasoft pseudopotentials to the projector augmented-wave method. Phys. Rev. B 59, 1758-1775 (1999).

41. Kresse, G. \& Furthmüller, J. Efficient iterative schemes for ab initio total-energy calculations using a plane-wave basis set. Phys. Rev. B 54, 11169-11186 (1996).

42. Kresse, G. \& Furthmüller, J. Efficiency of ab-initio total energy calculations for metals and semiconductors using a plane-wave basis set. Comput Mater. Sci. 6, 15-50 (1996).

43. Gao, Y., Shao, N., Pei, Y., Chen, Z. \& Zeng, X. C. Catalytic activities of subnanometer gold clusters $\left(\mathrm{Au}_{16}-\mathrm{Au}_{18}, \mathrm{Au}_{20}\right.$, and $\left.\mathrm{Au}_{27}-\mathrm{Au}_{35}\right)$ for $\mathrm{CO}$ oxidation. ACS Nano 5, 7818-7829 (2011).

44. Aprà, E. \& Fortunelli, A. Density-functional calculations on platinum nanoclusters: $\mathrm{Pt}_{13}, \mathrm{Pt}_{38}$, and $\mathrm{Pt}_{55}$. J. Phys. Chem. A 107, 2934-2942 (2003).

45. Henkelman, G. \& Jónsson, H. Improved tangent estimate in the nudged elastic band method for finding minimum energy paths and saddle points. J. Chem. Phys. 113, 9978-9985 (2000)

46. Weinan, E., Ren, W. \& Vanden-Eijnden, E. Simplified and improved string method for computing the minimum energy paths in barrier-crossing events. J. Chem. Phys. 126, 164103 (2007).

47. Weinan, E., Ren, W. \& Vanden-Eijnden, E. String method for the study of rare events. Phys. Rev. B 66, 52301 (2002).

\section{ACKNOWLEDGEMENTS}

Computations were performed in part using supercomputing resources at the National Energy Research Scientific Computing Center (NERSC). NERSC is supported by the U.S. Department of Energy, Office of Science, under contract DE-AC0205CH11231. We thank Ahmed Elnabawy and Lang Xu for their helpful comments on this manuscript. Work at UW-Madison was supported by Department of Energy-Basic Energy Sciences, Division of Chemical Sciences (grant DE-FG02-05ER15731). B.W.J.C. was partially supported by an Agency for Science, Technology, and Research (A*STAR) Singapore fellowship.

\section{AUTHOR CONTRIBUTIONS}

M.M. conceived and supervised the project. T.S. developed the concept of the algorithm, performed calculations and data analysis; B.W.J.C. implemented the algorithm and code for analysis of data and performed calculations and data analysis.
All authors discussed and wrote the manuscript. T.S. and B.W.J.C. contributed equally to this work.

\section{COMPETING INTERESTS}

The authors declare no competing interests.

\section{ADDITIONAL INFORMATION}

Supplementary information is available for this paper at https://doi.org/10.1038/ s41524-019-0240-x.

Correspondence and requests for materials should be addressed to M.M.

Reprints and permission information is available at http://www.nature.com/ reprints

Publisher's note Springer Nature remains neutral with regard to jurisdictional claims in published maps and institutional affiliations.

(i) Open Access This article is licensed under a Creative Commons Attribution 4.0 International License, which permits use, sharing, adaptation, distribution and reproduction in any medium or format, as long as you give appropriate credit to the original author(s) and the source, provide a link to the Creative Commons license, and indicate if changes were made. The images or other third party material in this article are included in the article's Creative Commons license, unless indicated otherwise in a credit line to the material. If material is not included in the article's Creative Commons license and your intended use is not permitted by statutory regulation or exceeds the permitted use, you will need to obtain permission directly from the copyright holder. To view a copy of this license, visit http://creativecommons. org/licenses/by/4.0/.

(c) The Author(s) 2019 